\title{
Erratum to: Euro-Par 2017: Parallel Processing Workshops
}

Dora B. Heras, Luc Bougé, Gabriele Mencagli, Emmanuel Jeannot, Rizos Sakellariou, Rosa M. Badia, Jorge G. Barbosa, Laura Ricci, Stephen L. Scott, Stefan Lankes, and Josef Weidendorfer

\section{Erratum to:}

D. B. Heras and L. Bougé (Eds.):

Euro-Par 2017: Parallel Processing Workshops, LNCS 10659, https://doi.org/10.1007/978-3-319-75178-8

The original version of the Book Frontmatter has been revised: In addition to the first two volume editors the seven Workshop Chairs have been added as volume editors. 